\title{
Rotating Biological Contactor for Biological Treatment of Poultry Processing Plant Wastewater Using Saccharomyces Cerevisiae
}

\author{
Dr. Ghasem D Najafpour, \\ Punita Nook Naidu and \\ Azlina Harun Kamaruddin \\ Associate Professor, School, Universiti Sains Malaysia, Engineering Campus \\ 14300 Nibong Tebal, S.P.S. Pulau Pinang \\ Tel: $00604-5937788$ ext. 5404,Fax: 00604 -594 1013,najafpour@hotmail.com
}

\begin{abstract}
Biological treatment using attached growth on a rotating biological contactor (RBC) was implemented for wastewater from poultry industries, which contains a high level of organic compounds due to the slaughtering, rendering of bones and fats, and plucking processes. The wastewater mostly consists of proteins, blood, fat and feathers. Nutrients available in the wastewater may enhance the growth of microorganisms, thus, it allows biological treatment to be used effectively. On the other hand, there are problems associated with biological treatment processes such as nutrients promoting algae growth. The control variables for the attached growth in $\mathrm{RBC}$ were wastewater detention time, effective surface for attached growth, disc submerged level in the wastewater basin, shaft rotation for aeration, $\mathrm{pH}$ control and supplementary nutrients. The attached growth resulted to high COD removal. The best treatment was obtained after 24 hours with $29 \%$ disc submergence level. The effective surface area for cell growth was $10.7 \mathrm{~m}^{2}$ using 60 discs mounted on a shaft. The microorganism used for the attached microbial growth was Saccharomyces cerevisiae. The shaft was rotating at $11 \mathrm{rpm}$. The treatment was improved with the addition of $1 \mathrm{v} / \mathrm{v}$ of 0.1 molar sodium hydroxide solution to the wastewater for controlling $\mathrm{pH}$. A $91 \%$ COD removal was obtained with $\mathrm{RBC}$ operating at optimum conditions, with a DO concentration of $3.98 \mathrm{mg} / \mathrm{l}$.
\end{abstract}

Key words: Rotating biological contactors, Aerobic treatment, Poultry processing wastewater, Saccharomyces cerevisiae, Attached growth.

\section{INTRODUCTION}

Rotating biological contactor $(\mathrm{RBC})$ is also popularly known as rotating bio-disc contactor. $\mathrm{RBC}$ contains a basin filled with wastewater with closely spaced discs mounted on a horizontal shaft above the wastewater tank. The discs are mounted perpendicularly to the flow of wastewater and a microbial growth is attached to one of the disc.
The surface of submerged discs in the wastewater is in the range of 25 to $40 \%$ (Scott and Smith, 1983). The selection of the bio-disc material is important because biofilm growth varies with different types of materials (Apilanez et al. 1998). When the disc with the attached microbial growth slowly rotates, it carries a thin film of wastewater to the atmosphere whereby microorganisms on the disc will then use oxygen in the atmosphere 
to treat the nutrients in the wastewater (Davis and Cornwell, 1991). The RBC used in this research has the capability to retain a large amount of biomass on the surface of the discs. Aside from that, the RBC has the ability to withstand hydraulic and organic surges (Canale, 1971).

One of the important parameters which has to be considered in the scale of $\mathrm{RBC}$ is mass transfer coefficient for oxygen. The experimental value for oxygen transfer coefficient has been determined (Kim and Molof, 1982) and is in the range of 49 to $87 \%$ theoretical value. The value of dissolved oxygen in $\mathrm{RBC}$ process is proportional to extended aeration surface and disc rotational speed. The performance of $\mathrm{RBC}$ has been demonstrated by other investigators, it was found to be highly efficient in operating at ambient temperature (Wilson and Meng, 1997).

A properly designed $\mathrm{RBC}$ is very reliable based on the amount of biomass attached to the discs. The biomass permits the RBC to more effectively withstand hydraulic and organic surges. The hydraulic characteristic of a bench scale model is determined by liquid flow rate, the rotational speed of the disc and the percentage of submerged surface (Banerjee, 1998).

Wastewater sample for treatment was obtained from Dindings Poultry Processing Plant in Sitiawan, Perak, Malaysia. The sample was taken to the influent point of the wastewater treatment system immediately after a rotary screening was done where large solid chunks such as feet, heads and feathers were removed. The plant processeses up to 40,000 birds everyday to produce chicken meat, chicken balls and nuggets. The plant discharges $1000 \mathrm{~m}^{3}$ of wastewater every day.

In this research, the optimum operational parameters for the fabricated $\mathrm{RBC}$ were found for the treatment of the Dindings Poultry Processing Plant's wastewater.

\section{MATERIALS AND METHODS}

The bench scale $\mathrm{RBC}$, a rectangular tank, was fabricated from an acrylic plastic transparent sheet with a thickness of $10 \mathrm{~mm}$, a length of 80 $\mathrm{cm}$, a width of $40 \mathrm{~cm}$, and a depth of $30 \mathrm{~cm}$. A transparent material was used to build the tank in order to observe wastewater throughout the experiment. The material was thick enough to withstand wastewater working volume pressure on the tank walls. A galvanized hollow metal bar with a diameter of $1.93 \mathrm{~cm}$ was used as the shaft. A metal bar was used to support the weight of the discs and the attached microbial growth as cell growth, was increased during each experimental run. The disc was made from acrylic plastic plate which is $1.5 \mathrm{~mm}$ thick and has a diameter of 35 $\mathrm{cm}$. Two sets of discs were fabricated with 40 and 60 discs. For each set useful area available for attached growth were 7 and $10.7 \mathrm{~m}^{2}$. Acrylic sheet was chosen because it is light weight and it does not react with microbial growth. In order to extend the surface area of each disc for efficient treatment, acrylic extended plates with a thickness of $1.5 \mathrm{~mm}$, a length of $12 \mathrm{~cm}$ and a width of $3 \mathrm{~cm}$ were attached to the discs. This model is designed and fabricated for batch mode operation.

Saccharomyces cerevisiae, a strain of yeast, was used as the attached microbial growth on RBC. S. cerevisiae was selected because it grows fast, can be obtained in bulk, easily adaptable to a new habitat and, safe to handle (Campbell and Duffus, 1988). Prior to the start of an experimental run, a $3 \%$ agar solution was evenly sprayed on both sides of the discs. The seed culture of $S$. cerevisiae was grown separately in a medium which consisted of glucose, peptone and yeast extracts in 3,1 and $1 \mathrm{~g} / 1$ respectively in a $250 \mathrm{~mL}$ flask. The 24 -hour incubated cultured medium was used as an inoculum for a larger volume and was introduced into the system. After a biofilm of microorganisms was developed on the thin layer of agar, an additional 24-hour incubation secured the system for sufficient cell growth. Then the $\mathrm{RBC}$ was ready to start an experimental run. The RBC was operated for five days using 60 liters of wastewater from Dindings Poultry Processing Plant and 1 liter of $S$. cerevisiae was used as a starter. Throughout the experiments, five major parameters have been observed, based on the Standard Methods for the Examination of Water and Wastewater. These are: total solids, total suspended solids, total Kjeldahl nitrogen, chemical oxygen demand (COD) and dissolved oxygen (DO). For COD analysis, the closed reflux calorimetric method was developed using dichromate reagent. The COD calibration curve was prepared using potassium hydrogen phthalate standard solution. A spectrophotometer (Milton Roy Spectronic 20D) at $600 \mathrm{~nm}$ was used to measure the absorbance of samples 
analyzed for COD. While for Total Kjeldahl Nitrogen (TKN), a semi-micro Kjeldahl method was used. All other routine tests were carried out based on instructions given in the Standard Methods for the Examination of Water and Wastewater (Greenberg et al., 1992).

The cell optical density was measured by light absorbance at $520 \mathrm{~nm}$ using a spectrophotometer. The dissolved oxygen concentration in wastewater was measured in $\mathrm{mg} / \mathrm{l}$ by a DO meter. The DO meter was WTW DO Cell OX 325 electro DO probe, Germany.

\section{RESULTS AND DISCUSSION}

Rotating biological contactors used in all experimental runs were loaded with induced biofilm of $S$. cerevisiae on the agar-coated surface of contacting discs. The fast growing microorganisms enhanced the treatment and reduced the hydraulic retention time. The RBC with induced biofilm of $S$. cerevisiae was quickly stabilized then used for wastewater treatment. The advantage of bench scale RBC was to conduct series of experimental parameters which were influencing on the biological treatment. The influent wastewater samples taken from the poultry processing plant were characterized. Several batches of wastewater samples were taken at different times of operation and had been analyzed. The characteristic of poultry processing wastewater has been summarized with the analysis of several samples. An average COD concentration of 2200 , total solids (TS) of $500-1500$, total suspended solids (TSS) of 650 , TKN of $15-25$, and DO of $0.3 \mathrm{mg} / \mathrm{l}$ were obtained based on experimental data resulting from the analysis.

The fabricated bench scale $\mathrm{RBC}$ was used for the treatment of poultry processing wastewater. Attached growth of microbial film of $S$. cerevisiae was developed on the surface of discs for an incubation period of 24 hours. A fixed volume of 60 liters wastewater was used. Mounted disc shafts with a sufficient load of biomass on 40 and 60 discs were rotated for 13 and $11 \mathrm{rpm}$, respectively. One $\mathrm{v} / \mathrm{v} \%$ of $0.1 \mathrm{M}$ sodium hydroxide solution was used for the pretreatment stage. Eight sets of experimental runs were selected to represent major parameters affected on RBC.

The wastewater for runs $36,38,42$ and 47 were treated for 96 hours. The experimental runs of $50,54,57$ and 60 were carried out in shorter period of 24 hours. All experimental runs has an effective surface area of $7 \mathrm{~m}^{2}$ except runs 57 and 60 , where the surface area for the extra load of biomass was increased by $53 \%$. Initially, the discs submergence level was $35 \%$ and the rotation was $13 \mathrm{rpm}$. Modification for improved shafts with extra discs resulted to the submergence level being reduced to $29 \%$ and the rotation to $11 \mathrm{rpm}$.

The treatment of wastewater was monitored based on COD reduction and cell optical density. Since the process is aerobic, the DO concentration was detected in the entire treatment process. The constant DO level, which was in a reasonable range, represents the system being sufficiently aerated and the growth of the biofilm on the discs proved that the attached growth occurred while the biofilm was exposed to the air. If the DO level was very low, this showed that the system had a deficiency for aeration. In contrast, at high levels of DO, the biomass growth was enhanced because the activity of microorganisms exponentially developed. Figure 1 shows the last data points for DO concentrations were $6,5,6.7$ and $7 \mathrm{mg} /$ 1 for runs 36, 38, 42 and 47 , respectively. The DO level for 29 and $35 \%$ was compared, as the discs submergence level was $6 \%$ lowered, the DO concentration was higher since the discs surface exposed to air was also higher. The maximum microbial activities based on cell optical density was experimentally determined in wastewater treatment at 24 hours, therefore, the next set of experiments were carried out for shorter periods of treatment. More than $70 \%$ of the cell optical density dropped after 48 hours of treatment due to nutrient depletion and/or the development of inhibitors in the wastewater. Biomass activities and cell growth rate are directly related to the $\mathrm{DO}$ level in the wastewater.

DO concentration was increased with the addition of $1 \mathrm{v} / \mathrm{v} \%$ of 0.1 molar sodium hydroxide solution. This phenomenon was due to chemical activities and the shift of $\mathrm{pH}$ from 6 to 8.5 which improved the solubility of oxygen and decreased microbial growth activities. The ethanol producer $S$. cerevisiae has maximum activities at around $\mathrm{pH}$ of 4.5-5.0. Since microbial growth was suppressed, the DO level has increased. The cell density of S. cerevisiae was drastically decreased after 48 hours of incubation and fast growing microorganisms reached their death phase. 


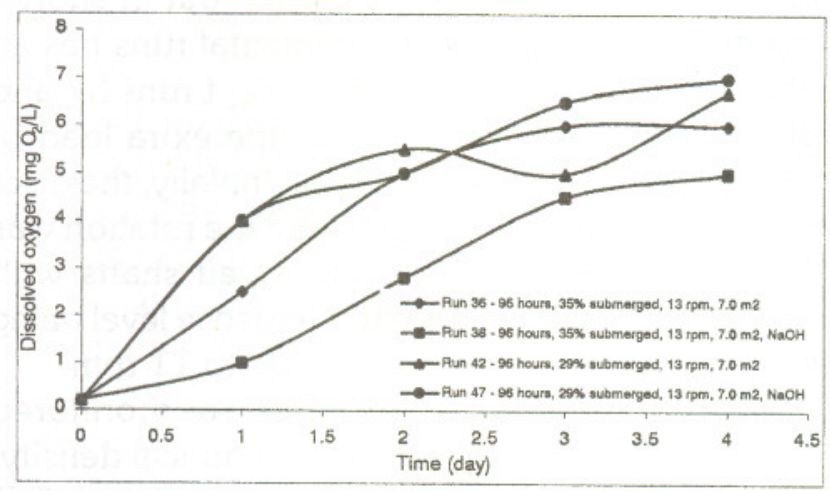

Figure 1: The DO level in the treated wastewater for runs $36,38,42$ and 47

Figure 2 shows the DO levels in the treated wastewater for 24 hours, 2.8, 3.0, 5.0 and 4.0 $\mathrm{mg} / \mathrm{l}$ for runs $50,54,57$ and 60 , respectively. A comparison of DO levels for runs 50 and 60 shows that DO level increased by about $50 \%$ as the disc surface exposed to the air increased. Prolonging wastewater treatment duration for four days may not improve treatment since $S$. cerevisiae and other microbial poputations drastically decreased after 48 hours of treatment. The duration of treatment caused ammonia

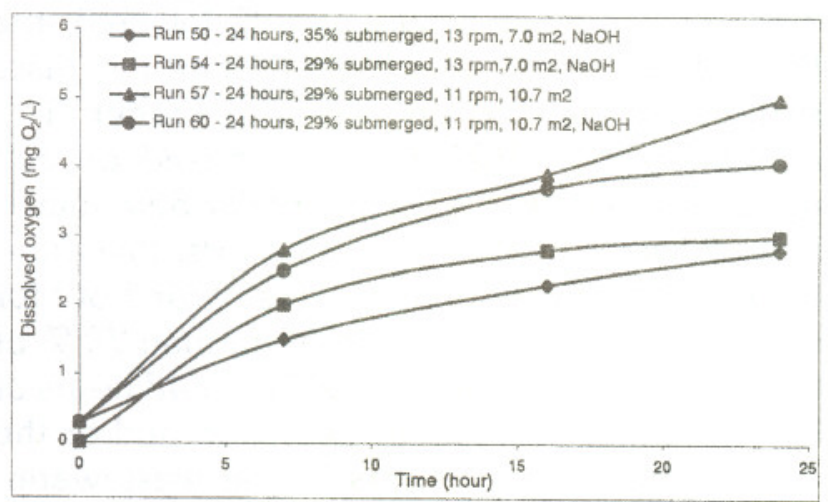

Figure 2: The DO levels in the treated wastewater for runs 50, 54, 57 and 60

build-up in the treatment system. In addition, the long period of treatment may also caused all microorganisms in the treated wastewater to go through endogenous phase since nutrient was depleted. Figure 3 shows the general trend of ammonia build up in most experimental runs as microbial activities declined. The minimum TKN concentration was at 24 hours of treatment. The results obtained from six experimental runs that used batches of wastewater sample, comparatively showed the same trend. The DO showed a constant increment ranging from 5 to 6 $\mathrm{mg} \mathrm{O}_{2} / \mathrm{l}$. On the other hand, the TKN showed reductions ranging from 50 to $65 \%$ by 24 hours due to nitrification whereby ammonia-nitrogen is converted to nitrites and nitrates.

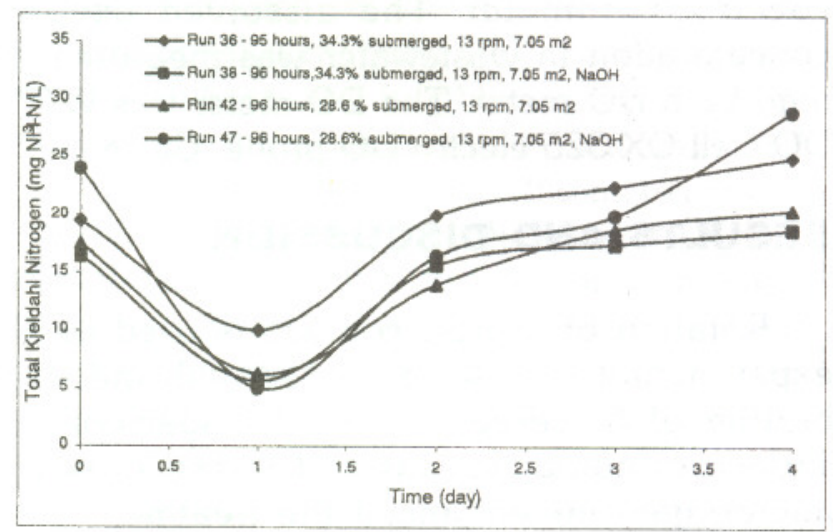

Figure 3: TKN concentrations in the treated wastewater for runs 36, 38, 42 and 47

Figure 4 shows the reduction of TS, TSS and COD. TS and COD had showed steady removal rate of 65 to $80 \%$ and 70 to $90 \%$, respectively. Meanwhile, TSS exhibited a sinusoidal trend. The TSS recorded a removal rate of about $80 \%$ for a total retention time of 96 hours. The TSS reduction within 24 hours, which ranged from 60 to $77 \%$, occurred due to suspended organic matters used by microbes for metabolism. A sudden increase in TSS from 24 hours to 72 hours was probably initiated by the high death rate of $S$. cerevisiae and other microorganisms. When nutrients were totally depleted after 72 hours, the amount of TSS decreased again.

Basically, the trend shown in Figures 3 and 4 were due to the slow rotation of the shaft, the low submergence level of the disc in the wastewater where a large part of the disc is exposed to the atmosphere, and the large surface area for treatment. These factors contributed to a high aeration rate in the system. Thus, the COD level was effectively reduced while DO was kept at a satisfactory level.

The low rotation of $11-13 \mathrm{rpm}$ and low shear forces did not cause any turbulence to break solid flocs. Therefore, TS and TSS were effectively reduced. Aerobic conditions and the existence of nitrification bacteria and Nitrobacter in the system caused the nitrification process. These species of 


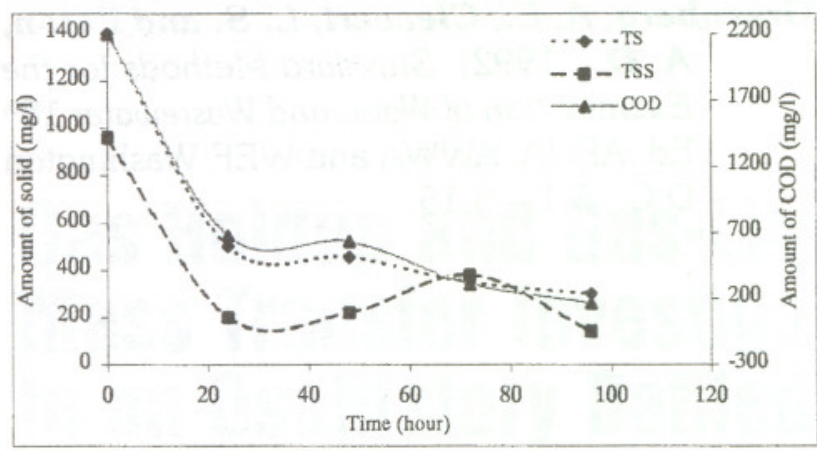

Figure 4: Efficiency of treatment observed through TS, TSS and COD

microbes, also known as Nitrifiers, converts ammonia nitrogen to nitrites and nitrates, which reduces TKN level. A sudden increase in TKN level was probably due to depletion of Nitrifiers. Certain modes of toxicity for Nitrifiers also occurred causing the TKN to drop. This is due to the sudden high concentration level of nitrites. A high concentration of nitrites and ammonia may cause toxicity for Nitrifiers. Nitrite concentration have increased due to the doubling of $\mathrm{DO}$ concentration after 24 hours and onwards.

Figure 5 shows COD reduction for runs 36 , 38,42 and 47 with a fixed disc surface of $7 \mathrm{~m}^{2}$. The final COD values were less than $400 \mathrm{mg} / \mathrm{l}$. The rate of COD removal for runs $36,38,42$ and 47 were $78,72,88$ and $94 \%$, respectively. The higher rate of COD removal for runs 42 and 47 compared to runs 36 and 38 were due to better aeration as $6 \%$ more of the surface area of the discs were exposed to the air. However, the addition of chemical did not have any positive effect on COD removal. Based on data available the reduction of COD was $70 \%$ within 24 hours.

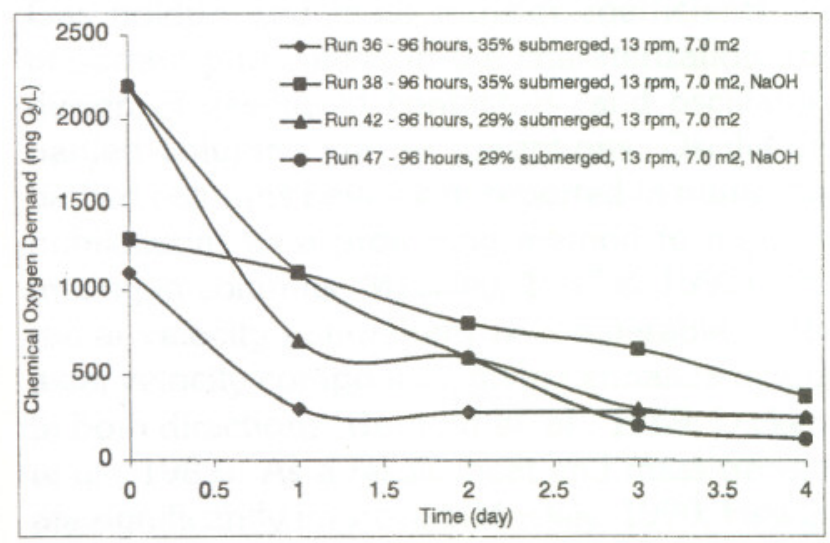

Figure 5: $C O D$ concentration reductions in wastewater for runs 36, 38, 42 and 47
The effect of additional surface area of disc and more surface exposure to air is shown in Figure 6. By comparing runs 50 and 54, the additional $6 \%$ surface exposure to air showed that COD reduction in the 24-hour wastewater treatment improved from 60 to $75 \%$. The use of a higher disc surface area of $10.7 \mathrm{~m}^{2}$, which is 53 $\%$ higher, improved the rate of COD removal from 60 to $90 \%$ and 75 to $92 \%$ in experimental runs 57 and 60, respectively. The DO level in the wastewater for experimental run 60 was about 4 $\mathrm{mg} / \mathrm{l}$ and the TKN removal rate was $75 \%$ after 24 hours of treatment. Addition of S. cerevisiae as attached growths on the RBC system enhanced the treatment process and shorten the duration of the treatment since fast growing bacteria was incorporated. A 24-hour treatment showed high achievement.

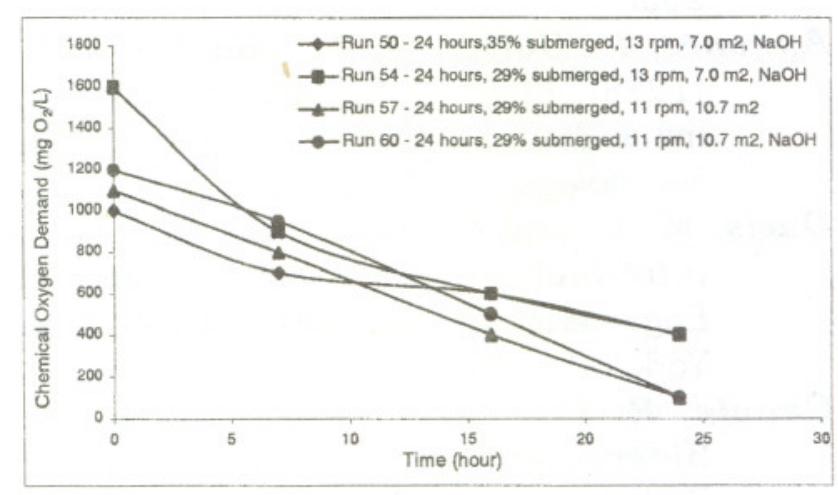

Figure 6: COD concentration reductions in wastewater for Run 50, 54, 57 and 60

\section{CONCLUSION}

This research concludes that the bench scale fabricated RBC model using an attached film of S. cerevisiae is best during a retention time of 24 hours, since TKN value was at its lowest level at this point. But in order to improve TS, TSS and COD removal rate, shaft rotation speed must be further reduced. In addition, discs surface area for the treatment must be extended or more discs must be added. Runs must also be based on optimum surface loading. In the latter stage of this research, the $\mathrm{RBC}$ bench model was modified based on the primary results gathered to optimize the operational parameters for effective aeration and the design parameters for efficient wastewater treatment. Application of $S$. cerevisiae as a film of fast-growing biomass on disc surface reduced 
treatment period of the poultry processing wastewater to about one day.

\section{ACKNOWLEDGEMENT}

The authors would like to acknowledge the Research and Development Committee of the Universiti Sains Malaysia for funding the research based on IRPA grant No. 0735115. We also like to thank the Dindings Poultry Processing Plant in Sitiawan, Perak State, Malaysia for their cooperation.

\section{REFERECENCES}

Scott, J. S. and Smith, P. G., (1983). Dictionary of Waste and Water Treatment, Butterworth, London. Scott and Smith, 262.

Apilanez, I., Gutierrez, A., and Diaz, M., (1998). Effect of Surface Materials on Initial Bioflim Development, Bioresource Technology: 66 (3), 225-230.

Davis, M. L. and Cornwell, D. A., (1991). Introduction to Environmental Engineering 2 $2^{\text {nd }}$ Ed., McGraw-Hill, New York, $368-370$.

Canale, R. P., (1971). Biotechnology and Bioengineering Symposium No. 2: Biological Waste Treatment, John Wiley \& Sons, New York, 131 - 140.

Kim, B. J. and Molof, A. H., (1982). The Scale up and Limitation of Physical Oxygen Transfer in Rotating Biological Contactors, Water Science and Technology: 14, 569579.

Wilson, F. and Meng, L. W., (1997). Rotating Biological Contactors for Wastewater Treatment in an Equatorial Climate, Water Science and Technology: 35 (80), $177-$ 184.

Banerjee, G., (1998). Hydraulics of Bench Scale Rotating Biological Contactor. Water Research: 31 (10), 2500-2510.

Campbell, I. and Duffus, J. H., (1988). Yeast: A Practical Approach. IRL Press, Oxford, Washington D.C., 1 - 5 .
Greenberg, A. E., Clesceri, L. S. and Eaton, A. D., (1992). Standard Methods for the Examination of Water and Wastewater $18^{\text {th }}$ Ed. APHA, AWWA and WEF, Washington D.C., $5.1-5.15$. 